\title{
Genetic influences contributing to LDL particle size in familial combined hyperlipidaemia
}

\author{
Juha Vakkilainen ${ }^{1,8}$, Päivi Pajukanta ${ }^{2,8}$, Rita M Cantor ${ }^{2,3}$, Ilpo O Nuotio ${ }^{4,5}$, \\ Sanni Lahdenperä ${ }^{1}$, Kati Ylitalo ${ }^{1}$, Jussi Pihlajamäki ${ }^{6}$, Petri T Kovanen ${ }^{7}$, Markku Laakso $^{6}$, \\ Jorma SA Viikari $^{5}$, Leena Peltonen ${ }^{2}$ and Marja-Riitta Taskinen ${ }^{* 1}$
}

\begin{abstract}
${ }^{1}$ Department of Medicine, Helsinki University Central Hospital, Helsinki, Finland; ${ }^{2}$ Department of Human Genetics, UCLA, Los Angeles, CA, USA; ${ }^{3}$ Department of Pediatrics, UCLA, Los Angeles, CA, USA; ${ }^{4}$ Cardiovascular Research Unit, Turku University Central Hospital, Turku, Finland; ${ }^{5}$ Department of Medicine, Turku University Central Hospital, Turku, Finland; ${ }^{6}$ Department of Medicine, Kuopio University Hospital, Kuopio, Finland; ${ }^{7}$ Wihuri Research Institute, Helsinki, Finland
\end{abstract}

The nature of the genetic and environmental factors influencing low density lipoprotein (LDL) particle size in patients with familial combined hyperlipidaemia (FCHL) is under debate. We measured LDL peak particle size in 553 subjects belonging to 48 Finnish FCHL families. Individuals with high triglyceride (TG) concentrations (phenotype IV) or combined hyperlipidaemia (phenotype IIB) had significantly smaller LDL particles than those with hypercholesterolaemia (phenotype IIA) or unaffected subjects $(P<0.001)$. In stepwise regression analyses, serum TGs $\left(r^{2}=43 \%, P<0.001\right)$ and high density lipoprotein cholesterol (HDL-C) $\left(r^{2}=4.5 \%, P<0.001\right)$ were the only significant predictors of LDL peak particle size. Familial correlations support the conclusion that LDL peak particle size is familial, and most probably influenced by genes in these families. Segregation analysis of LDL peak particle size, a quantitative trait, was performed to model this genetic influence. Our results suggest a polygenic background for LDL size with a recessive major gene that may contribute to large LDL peak particle size in women. Serum TG and HDL-C concentrations predict the majority of variations in LDL particle size.

European Journal of Human Genetics (2002) 10, 547-552. doi:10.1038/sj.ejhg.5200844

Keywords: Small dense LDL; segregation analysis; hypertriglyceridaemia; low HDL cholesterol; dyslipidaemia

\section{Introduction}

Familial combined hyperlipidaemia (FCHL) is a common hereditary dyslipidemia with a prevalence of $1-2 \%$. It is characterised by elevated levels of serum total cholesterol (TC), triglycerides (TGs), or both. ${ }^{1,2}$ Decreased high density lipoprotein cholesterol (HDL-C) levels are also frequently found in FCHL, especially in association with high TG values. ${ }^{3}$ Possible defects in FCHL are proposed to be the

\footnotetext{
*Correspondence: Prof. Marja-Riitta Taskinen, Helsinki University Centra Hospital, Department of Medicine, Haartmaninkatu 4, PO Box 340, 00029 HUCH, Helsinki, Finland. Tel: +358-9-47172240; Fax: +358-9-47174694; E-mail: mataskin@helsinki.fi

${ }^{8}$ The authors have contributed equally to the work contained in this manuscript

Received 19 December 2001; revised 23 April 2002; accepted 16 May
} 2002 overproduction and impaired clearance of apolipoprotein B (apoB) containing lipoproteins. ${ }^{4}$ Both environmental and genetic factors are suggested to affect the complex FCHL phenotype, ${ }^{5}$ although the precise metabolic and genetic defects responsible for FCHL are still not known.

Low density lipoprotein (LDL) particles are heterogeneous. LDL subclasses can be differentiated according to the density or the electrophoretical movement translated into the terms of the particle size. ${ }^{6,7}$ LDL particle size classification has been of interest because of identified associations between small, dense LDL particles and coronary heart disease (CHD). ${ }^{8}$ In addition, the LDL subclass distribution has been associated with serum TG and HDLC concentrations, ${ }^{9}$ TC to apoB ratio, ${ }^{9}$ hepatic lipase (HL) activity, ${ }^{10}$ and insulin resistance. ${ }^{11}$ 
Previous studies implicated genetic susceptibility and environmental factors in the expression of LDL particle size and density in FCHL families. ${ }^{12,13}$ A recent study supported the strong association between small LDL particles, high serum TGs, and low HDL-C in FCHL and familial hypertriglyceridaemia families, and it was inferred that the same gene(s) affect all three traits. ${ }^{14}$ Metabolic associations between high serum TG concentrations, low HDL-C concentrations, and small dense LDL are well established. ${ }^{9,15}$ The purpose of the current study was to add to our understanding of the regulation of LDL peak particle size in FCHL.

\section{Materials and methods Subjects}

The study sample consisted of 48 Finnish FCHL families $(n=720)$. The study protocol has been described in detail elsewhere. ${ }^{3}$ Affection status for family members was based on the age and sex specific 90th percentile levels of serum TC and TGs, derived in the general Finnish population, as described earlier. ${ }^{16,17}$ Subjects using lipid-lowering medication were asked to discontinue the medication for 30 days before collection of blood samples, which were taken after a $10-12 \mathrm{~h}$ overnight fast. All traits were measured on 553 individuals: 441 family members and 112 spouses. The study was approved by the ethics committees of the participating centres, and all participants gave their informed consent.

\section{LDL peak particle size and other measurements}

LDL peak particle diameters, serum TC, TGs, HDL-C, and apoB were determined as described earlier. ${ }^{18,19}$ TC values were transformed to $\mathrm{g} / \mathrm{l}$ values in the $\mathrm{TC} / \mathrm{apoB}$ ratios by dividing the TC values by 2.6. The lipid values of each subject were determined in one to three visits within short intervals. The maximum of all available values was used except for HDL-C and TC/apoB ratios, for which the minimum was used. Body mass index (BMI) was calculated as weight $(\mathrm{kg}) /$ height $(\mathrm{m}){ }^{2}$

\section{Statistical analyses of traits}

Lipid characteristics of different FCHL phenotypes were compared by analysis of variance (ANOVA). Scheffe's (equal variances) and Tamhane's (non-equal variances) post hoc tests were conducted when appropriate. The differences between groups with different lipid phenotypes were tested with a two-way ANOVA using the phenotype as a fixed factor and family membership as a random factor. ${ }^{20}$ Family number was also incorporated in regression analyses as a factor to adjust for the non-independence of family members. Stepwise regression was performed to study the relationships among traits correlated with LDL particle size. Spouses were excluded from these analyses. Statistical tests were performed with SPSS 8.0 (SPSS Inc., Chicago, IL, USA). A $P$-value $<0.05$ was considered significant. The data are shown as mean \pm SD. Natural logarithmic transforma- tions were applied to reduce skewness when appropriate. No transformation of the LDL size trait was made.

\section{Analyses of family data}

Familial correlations were estimated using the FCOR2 program of SAGE. ${ }^{21}$ The uniform weight to pedigrees option was chosen. This programme estimates correlations among relatives taking into account the lack of independence among family members in assessing the statistical significance. Prior to analysis, adjustments for age and gender on LDL size were done by regression analysis, and correlations were conducted on the residuals.

To extract the maximum amount of information, all traits were treated as quantitative. Segregation analyses of LDL peak particle size were performed using the REGC programme of SAGE. ${ }^{22}$ This programme follows the approach described by Bonney et al. ${ }^{23}$ Trait distributions are generated by conditioning each individual's value on that of his antecedents. The class A models, used here, assume that the dependency of the trait among siblings can be explained solely by their common parentage. The models do not contain a separate component for shared environment. This can be considered reasonably appropriate for these Finnish families which are culturally more homogeneous than families not originating from isolated populations. Multivariate normality of the trait in the relatives is assumed, and the correlation structure among the relatives is explained by models which include parameters representing the effects of a major gene, polygenes, gender and other covariates. Parameters for the mean and standard deviation of each major genotype, as well as the residual correlations among relatives after the major gene is modelled are included. The major gene is also parametrised by its frequency and the mode of transmission. The polygenic model assumes mendelian inheritance and that the major gene has an allele frequency of zero. Table 3 shows the estimated parameters of the segregation analyses. Analyses were conducted by conditioning on the original FCHL proband in each family. We have not conditioned on the family members contributing to the diagnosis of FCHL. Many of them do not exhibit a particular LDL size profile, making this reasonable. Age and BMI were treated as covariates. When evaluating a particular segregation model, a statistic of negative two times the log likelihood ( -2 lnlik) of the data under that model with the 'best' parameter estimates is obtained. This statistic is used to compare the different segregation models in two ways. When two models are not nested, the likelihoods of these models are compared using Akaike's information criterion (AIC). ${ }^{24}$ The AIC is the -2 lnlik value with twice the number of independent estimated parameters added to it. When models are nested, the relative goodness of fit of the models is tested by calculating negative twice the difference in the $\log$ likelihoods which is distributed as $\chi^{2}$ with the difference in the degrees of freedom (equal to the number of independent parameters estimated by the two models). 


\section{Results}

Subject characteristics are shown in Table 1. The proportions of men and women were similar in all study groups. Fifty-one per cent of FCHL family members were unaffected, and they were younger and leaner than their affected relatives and spouses. LDL peak particle sizes were especially small in groups with high TGs (IIB and IV) $(P<0.001$ vs unaffected family members, IIA, and spouses). The prevalence of small LDL particles (LDL peak particle diameter $<25.5 \mathrm{~nm}$ ) was greatest in those with phenotype IIB or IV (Table 1). When LDL peak particle size was adjusted for age and BMI all three phenotypes had significantly smaller LDL particles than unaffected family members $(25.9 \pm 0.5,25.8 \pm 0.4,25.8 \pm 0.5$, and $26.4 \pm 0.5 \mathrm{~nm}$ for IIA, IIB, IV, and unaffected groups, respectively, $P<0.001$ for each).

In FCHL family members, serum TGs $(r=-0.66)$, TC/apoB ratio $(r=0.59)$, HDL-C $(r=0.53)$, apoB $(r=-0.51)$, TC $(r=-0.40)$, age $(r=-0.36)$, and BMI $(r=-0.35)$ were significantly correlated $(P<0.001$ for all $)$ with LDL particle size. In regression analyses using all 441 FCHL family members and adjusting for their non-independence, serum TGs and HDL-C were retained in a predictive model and explained 41.0 and $4.5 \%$ of LDL peak particle size variation, respectively. Other traits were not retained in this model due to their high correlations with these traits.
Familial correlations were estimated to assess the potential genetic contribution to LDL peak particle size (Table $2)$. The parent-offspring and sibling-sibling correlations exhibited values of 0.17 and $0.16(P \leqslant 0.01)$, whereas spousal correlations were non-significant, suggesting that LDL peak particle size in Finnish FCHL families is familial, and probably genetic, although the sample size may not be sufficient to detect a significant spousal correlation. Table 2 also shows the correlations of TC, TGs, apoB and HDL-C with LDL peak particle size. These data indicate that LDL peak particle size is familial, and appears to be correlated with TGs and HDL-C in family members, suggesting possible common genetic contributions to these traits.

To further assess the genetic contribution to LDL peak particle size, we conducted segregation analysis (Table 3). We began by testing a general transmission model with 12 free parameters, but unfortunately the model did not converge, although multiple sets of initial values were tried. However, we were able to fit more limited models. First, the frequency of a major gene allele in a mendelian model was set to 0 , resulting in one normal distribution capturing polygenic and random environmental effects (column 1 of Table 3). The addition of parameters for residual familial correlations (column 2) resulted in a significant improvement in the fit $(P<0.05)$, indicating that family members

Table 1 Subject characteristics

\begin{tabular}{|c|c|c|c|c|c|c|}
\hline & & FCHL family & members & & & \\
\hline & $\begin{array}{c}\text { Unaffected } \\
\mathrm{n}=224(121 \mathrm{~m} / 103 \mathrm{f})\end{array}$ & $\mathrm{n}=62(32 \mathrm{~m} / 30 \mathrm{f})$ & $\begin{array}{c}I / B \\
\mathrm{n}=78(42 m / 36 f)\end{array}$ & $\begin{array}{c}I V \\
\mathrm{n}=77(34 \mathrm{~m} / 43 \mathrm{f})\end{array}$ & P value & $\begin{array}{c}\text { Spouse } \\
\mathrm{n}=112(55 \mathrm{~m} / 57 \mathrm{f})\end{array}$ \\
\hline Age (years) & $32.1 \pm 12.8$ & $42.2 \pm 13.1^{*}$ & $43.7 \pm 13.9^{*}$ & $43.0 \pm 15.5^{*}$ & $<0.001$ & $50.9 \pm 8.4$ \\
\hline $\mathrm{BMl}\left(\mathrm{kg} / \mathrm{m}^{2}\right)$ & $24.1 \pm 3.9$ & $26.7 \pm 3.7^{*}$ & $27.8 \pm 3.4^{\star}$ & $28.0 \pm 4.2^{*}$ & $<0.001$ & $26.5 \pm 4.4$ \\
\hline LDL size $(\mathrm{nm})$ & $26.7 \pm 1.2$ & $26.2 \pm 1.3 \div$ & $25.3 \pm 1.5 * \dagger$ & $25.1 \pm 1.6^{*} \dagger$ & $<0.001$ & $26.7 \pm 1.3$ \\
\hline Prevalence of small $\mathrm{LDL}^{1}(\%)$ & $16.1 \div$ & $27.4 \div$ & $57.7^{*}+$ & $55.8 *+$ & $<0.001$ & $1 \overline{7.9}$ \\
\hline Total cholesterol $(\mathrm{mmol} / \mathrm{l})$ & $5.1 \pm 0.9$ & $7.3 \pm 0.8$ & $7.8 \pm 1.0$ & $6.0 \pm 0.8$ & & $5.9 \pm 1.0$ \\
\hline HDL cholesterol (mmol/l) & $1.25 \pm 0.32 \div$ & $1.40 \pm 0.43+\top$ & $1.08 \pm 0.38 * \dagger$ & $1.03 \pm 0.33 * \dagger$ & $<0.001$ & $1.32 \pm 0.41$ \\
\hline Triglycerides (mmol/l) & $1.26 \pm 0.54$ & $1.72 \pm 0.58$ & $4.43 \pm 2.90$ & $3.33 \pm 1.76$ & & $1.40 \pm 0.62$ \\
\hline Apolipoprotein B (apoB) $(\mathrm{g} / \mathrm{l})$ & $0.89 \pm 0.24+\div$ & $1.28 \pm 0.24^{*} *$ & $1.59 \pm 0.42 * \div$ & $1.23 \pm 0.25 *+$ & $<0.001$ & $1.03 \pm 0.24$ \\
\hline Total cholesterol/apoB ratio & $2.23 \pm 0.37+$ & $2.19 \pm 0.32 \div$ & $1.87 \pm 0.39 * \dagger$ & $1.88 \pm 0.34^{*} \dagger$ & $<0.001$ & $2.18 \pm 0.32$ \\
\hline
\end{tabular}

Data are mean \pm standard deviation. ${ }^{1} \mathrm{LDL}$ peak particle diameter $<25.5 \mathrm{~nm}$. ${ }^{2}$ Total cholesterol values transformed to $\mathrm{g} / \mathrm{l}$. ${ }^{3} \mathrm{One}$-way ANOVA for members in the FCHL family blood lines. Analyses adjusted for non-independence of family members. ${ }^{*} P<0.05$ vs Unaffected; $\uparrow P<0.05$ vs IIA; $\ddagger P<0.05$ vs IIB; $\uparrow P<0.05$ vs IV.

Table 2 Age-sex adjusted familial correlations among LDL peak particle size and TC, TGs, apoB, HDL-C

\begin{tabular}{|c|c|c|c|c|c|c|}
\hline Relative pairs & & LDL size & TC & $\begin{array}{l}\text { Trait } \\
\text { TGs }\end{array}$ & $a p o B$ & $H D L-C$ \\
\hline Spousal & LDL size of mother & -0.17 & $0.35(* *)$ & $0.28(* *)$ & $\left.0.311^{* *}\right)$ & 0.01 \\
\hline$(n=96)$ & LDL size of fathert & -0.17 & 0.19 & 0.17 & $0.23\left(^{*}\right)$ & -0.01 \\
\hline$(n=437)$ & LDL size of offspring $\$$ & $0.17\left(^{* *}\right)$ & -0.04 & $-0.17\left(^{(*}\right)$ & -0.10 & $0.11\left(^{*}\right)$ \\
\hline Sibling $(n=497)$ & LDL size & $0.16(* *)$ & 0.01 & $\left.-0.18{ }^{* *}\right)$ & -0.05 & $0.14\left(^{*}\right)$ \\
\hline
\end{tabular}

†Mother's LDL peak particle size correlation with father's other trait (LDL peak particle size, TC, TGs, apoB, or HDL-C). ††Father's LDL peak particle size correlation with mother's other trait (LDL peak particle size, TC, TGs, apoB, or HDL-C). $¥$ Parent's LDL peak particle size correlation with offspring's other trait (LDL peak particle size, TC, TGs, apoB, or HDL-C). HOOffspring's LDL peak particle size correlation with parent's other trait (LDL peak particle size, TC, TGs, apoB, or HDL-C). ${ }^{*} P<0.05 ;{ }^{* *} P<0.01$. 
Table 3 Segregation analysis of LDL peak particle size

\begin{tabular}{|c|c|c|c|c|c|c|}
\hline \multirow[b]{2}{*}{ Parameter } & \multicolumn{6}{|c|}{ Model* } \\
\hline & $\begin{array}{c}1 \\
\text { Sex-dependent } \\
\text { no major gene } \\
\text { no familial } \\
\text { correlations }\end{array}$ & $\begin{array}{c}2 \\
\text { Sex-dependent } \\
\text { no major gene } \\
\text { with familial } \\
\text { correlations }\end{array}$ & Dominant & $\begin{array}{l}\text { Sex-dependent } \\
\text { dominant }\end{array}$ & Recessive & $\begin{array}{l}\text { Sex-dependent } \\
\text { recessive }\end{array}$ \\
\hline 1. Frequency of major gene allele $A$ & 0 & 0 & $0.4 \times 10^{-6}$ & $0.8 \times 10^{-6}$ & $0.05(0.03)$ & $0.06(0.03)$ \\
\hline 2. Frequency of $A A$ & - & - & $0.1 \times 10^{-12}$ & $0.6 \times 10^{-12}$ & $0.002(0.003)$ & $0.004(0.004)$ \\
\hline 3. Frequency of $A B$ & - & - & $0.7 \times 10^{-6}$ & $0.2 \times 10^{-5}$ & $0.09(0.05)$ & $0.12(0.06)$ \\
\hline 4. Frequency of $B B$ & - & - & 1.0 & 1.0 & $0.90(0.05)$ & $0.88(0.06)$ \\
\hline & Female/Male & Female/Male & & Female/Male & & Female/Male \\
\hline 5. Mean AA & - & - & $3.5(1.4)$ & $23.0 / 3.2$ & $3.5(1.4)$ & $31.2 / 3.4$ \\
\hline 6. Mean $A B$ & - & - & $3.5(1.4)$ & $23.0 / 3.2$ & $28.9(0.4)$ & $29.3 / 28.5$ \\
\hline 7. Mean BB & - & - & $28.9(0.4)$ & $29.3 / 28.5$ & $28.9(0.4)$ & $29.3 / 28.5$ \\
\hline 8. Mean of Polygenic Distribution & $29.4 / 28.4$ & $29.4 / 28.4$ & - & - & - & - \\
\hline 9. Variance for each major gene distribution & $3.05(0.2)$ & $3.04(0.2)$ & $2.0(0.1)$ & $1.8(0.1)$ & $1.99(0.1)$ & $1.82(0.1)$ \\
\hline 10. Spouse Correlation & - & $-0.04(0.06)$ & $-0.20(0.08)$ & $-0.14(0.08)$ & $-0.20(0.08)$ & $-0.14(0.08)$ \\
\hline 11. Parent-Offspring Correlation & - & $0.10(0.04)$ & $0.18(0.04)$ & $0.20(0.04)$ & $0.18(0.04)$ & $0.20(0.04)$ \\
\hline 12. Regression Coefficient of Age & -0.015 & -0.015 & -0.009 & -0.01 & -0.009 & -0.01 \\
\hline 13. Regression Coefficient of $\mathrm{BMI}$ & -0.08 & -0.08 & -0.09 & -0.08 & -0.09 & -0.08 \\
\hline 14. Number of independent parameters & 5 & 7 & 8 & 10 & 8 & 10 \\
\hline 15. -2 Inlik & 2052 & 2045 & 1842 & 1796 & 1823 & 1774 \\
\hline 16. $\mathrm{AlC}^{* *}$ & 2062 & 2059 & 1858 & 1816 & 1839 & 1794 \\
\hline
\end{tabular}

${ }^{*}$ A general model with all parameters free to be estimated did not converge. ${ }^{* *}$ AIC indicates Akaike Information Criterion. Standard error (SE) values are shown in the parentheses. Model 2 vs model 1, $P<0.05$; Model 4 vs model 3, $P<0.0001$; Model 6 vs model $5, P<0.0001$

are correlated for the trait beyond that accounted for by polygenes. Further, the inclusion of a major gene resulted in a marked improvement in the fit. Modeling this gene was first done without considering the effects of gender (columns 3 and 5). However, impossible parameter estimates associated with the means and major gene allele frequencies of these models led us to continue by testing models that include gender effects. Models with gender effects (columns 4 and 6) added two extra parameters for female genotypic means (lines 5-7). Their inclusion improved the fit of both models significantly $(P<0.0001)$. However, only the estimated means of the female major genotypes were realistic. An additional model fixing the parameters for the residual spousal and parent-offspring correlations to zero for this recessive model with gender differences gave an AIC of 1800 (data not shown), indicating that these residual correlations contribute to the explanation of these data.

In conclusion, the estimated means and frequencies for the major genotypes of $\mathrm{AA}$ and $\mathrm{AB}$ for dominant and recessive models are unrealistic for all models except for the recessive one identified in women. This result suggests that the LDL size trait has a polygenic background with a recessive major gene that may contribute to large LDL peak particle size in women.

\section{Discussion}

We investigated the genetic contributions to LDL peak particle size in 48 FCHL families originating from the genetically isolated Finnish population, ${ }^{25}$ which offers advantageous study material for complex disorders such as
FCHL, as genetic and environmental homogeneity beyond that observed in more mixed populations are expected. Even in this homogeneous population, however, a polygenic model for LDL peak particle size is likely. A recessive, low frequency major gene may also contribute to LDL peak particle size in females.

Significant familial correlations of LDL peak particle size with TGs and HDL-C may indicate a common genetic background contributing to these traits. Correlations among LDL peak particle size, TGs, and HDL-C have also been identified in epidemiological studies conducted in general populations that have not been ascertained for disease ${ }^{9}$ as well as in FCHL families. ${ }^{13} \mathrm{~A}$ recent study inferred that in FCHL and familial hypertriglyceridaemia a large proportion of these correlations can probably be explained by the influence of at least one gene affecting all of these traits. ${ }^{14}$ However, non-shared environmental and possibly genetic factors also had a strong effect on them. These results could also be interpreted to imply that one or more shared genes has an effect on one of the traits, which in turn has secondary effects on the others. Accordingly, the primary dyslipidemic feature could be a high TG concentration leading to secondary changes in LDL size and HDL-C concentration. $^{26}$

Our quantitative segregation analysis of LDL peak particle size in 48 Finnish FCHL families suggests that this trait is the result of the additive effects of multiple genes where a recessive major gene of low frequency $(0.06 \%)$ may contribute to large LDL peak particle size in women. It is possible that this major gene is underrepresented among the hyperlipidaemic female FCHL family members 
when compared to the general female population, since hyperlipidaemic individuals tend to have small LDL sizes. The equal frequencies of men and women in these segregation analyses indicate that the same opportunity to identify a major gene for LDL size was available for both sexes. However, this sample may only have provided the information to identify the presence of this gene in females by chance alone. On the other hand, premenopausal women tend to have larger and less dense LDL particles than males of the same age, likely due to their lower hepatic lipase activity and lower serum TG concentration. ${ }^{9,10}$ These traits are regulated by genetic as well as other factors, such as obesity and sex hormone levels, suggesting that our finding reflects the genetic background and/or gene-environment interactions in these women.

There are limitations to interpreting the results of the segregation analyses, such as their dependence on the parameters used, models, method of analysis, and ascertainments. ${ }^{27}$ The relatively small study sample (553 subjects) indicates that inferences drawn from this study need to be conservative. However, our results may contribute to refining the complex FCHL phenotype for further genetic analyses. This is of especial interest, since it has recently been suggested that small dense LDL and apoB may be better candidates for defining the FCHL diagnosis ${ }^{28}$ when compared to the currently used serum TC and/or TG traits. ${ }^{1,2}$ The current study suggests that LDL size may not be such an attractive candidate for genetic studies, whereas several earlier studies suggested major gene effects for serum TG and apoB levels. ${ }^{29,30}$

Candidate genes for small, dense LDL have been examined intensively. Results of these studies have often been contradictory, and some of the positive results have not been confirmed. A recent study showed evidence indicating that several genes influence LDL size in FCHL, including lecithin: cholesteryl acyltransferase, manganese superoxide dismutase, and the apolipoprotein AI-CIII-AIV gene cluster. ${ }^{31}$ These data also lend support for a polygenic model for LDL particle size.

In a previous study, seven FCHL families consisting of 234 members showed that pattern B was inherited as an autosomal trait, with either a dominant or an additive mode of inheritance, and a small, but significant additional multifactorial component. ${ }^{12}$ In comparison to the current analyses, LDL particle size was treated as qualitative. This result was consistent with those of an earlier study of primarily healthy families, ${ }^{32}$ in which LDL particle size defined as a dichotomous trait fit a single dominant gene model of inheritance with reduced penetrance. However, when the same study sample was analysed using the LDL peak particle diameter as a continuous variable that was adjusted for age and gender, the models of inheritance could not be distinguished. ${ }^{33}$ Evidence for a recessive model of inheritance with an additional polygenic component was implicated for the small dense LDL trait in 40 Dutch FCHL families with 623 individuals, ${ }^{13}$ although in this study, unlike in the present one, the LDL subfractions were determined by LDL density, and not size. More recently, using those same families, a model containing a major gene with codominant alleles for both the LDL density and apoB traits, and distinct polygenes for each trait, was identified. ${ }^{34}$ Interestingly, in a study by Hokanson et al, lowering of serum TGs with gemfibrozil in FCHL patients resulted in a marked increase of LDL buoyancy, while LDL peak particle size remained small, ${ }^{35}$ suggesting that LDL particle size and buoyancy are regulated, at least in part, via different pathways. Analytic, methodological and population baseddifferences may account for these somewhat contradictory results.

In conclusion, small LDL particles are a typical feature of Finnish FCHL patients, found especially in individuals with phenotype IIB or IV. While we have not established that LDL peak particle size for men follows a strictly polygenic model, these analyses indicate that LDL size in men is unlikely to be influenced by a major gene in this population. In women, a low frequency recessive major gene allele may be contributing to larger LDL peak particle sizes. The observed correlations between LDL peak particle size, TGs, and HDL$\mathrm{C}$ may be due to a common genetic background and/or metabolic interplay.

\section{Acknowledgements}

We thank Seppo Sarna for statistical advice, and Helinä PerttunenNio, Ritva Marjanen, and Leena Lehikoinen for technical assistance. The results of familial correlations and segregation analyses were obtained by means of the program package SAGE which is supported by US Public Health Services Resource grant (1 P41 RR03655) from the National Center for Research Resources. This work was supported by grants from the Finnish Academy, Finnish Heart Foundation, Duodecim Foundation, Maud Kuistila Foundation, Helsinki University Central Hospital Research Foundation, and European Commission contract 'European Multicenter Study on Familial Dyslipidemias With Premature Coronary Heart Disease (EUFAM)', contract number BMH4-CT96-1678. P Pajukanta, RM Cantor and $L$ Peltonen were supported in part by the National Institutes of Health Program Project grant HL-28481.

\section{References}

1 Goldstein JL, Schrott HG, Hazzard WR, Bierman EL, Motulsky AG: Hyperlipidemia in coronary heart disease II. Genetic analysis of lipid levels in 176 families and delineation of a new inherited disorder, combined hyperlipidemia. I Clin Invest 1973; 52: $1544-1568$.

2 Nikkilä EA, Aro A: Family study of serum lipids and lipoproteins in coronary heart disease. Lancet 1973; 1: 954-958.

3 Porkka KVK, Nuotio I, Pajukanta P et al: Phenotype expression in familial combined hyperlipidaemia. Atherosclerosis 1997; 133: $245-253$.

4 de Graaf J, Stalenhoef AFH: Defects of lipoprotein metabolism in familial combined hyperlipidaemia. Curr Opin Lipidol 1998; 9: $189-196$.

5 Aouizerat BE, Allayee $\mathrm{H}$, Bodnar J et al: Novel genes for familial combined hyperlipidemia. Curr Opin Lipidol 1999; 10: 113-122. 
6 Krauss RM, Burke DJ: Identification of multiple subclasses of plasma low density lipoproteins in normal humans. J Lipid Res 1982; 23: $97-104$.

7 Austin MA, Hokanson JE, Brunzell JD: Characterization of lowdensity lipoprotein subclasses: methodologic approaches and clinical relevance. Curr Opin Lipidol 1994; 5: 395- 403.

8 Lamarche B, Tchernof A, Moorjani S et al: Small, dense lowdensity lipoprotein particles as a predictor of the risk of ischemic heart disease in men: prospective results from the Québec Cardiovascular Study. Circulation 1997; 95: 69-75.

9 McNamara JR, Campos H, Ordovas JM, Peterson J, Wilson PW, Schaefer EJ: Effect of gender, age, and lipid status on low density lipoprotein subfraction distribution. Results from the Framingham Offspring Study. Arteriosclerosis 1987: 483-490.

10 Tan CE, Foster L, Caslake MJ et al: Relations between plasma lipids and postheparin plasma lipases and VLDL and LDL subfraction patterns in normolipemic men and women. Arterioscler Thromb Vasc Biol 1995; 15: $1839-1848$.

11 Reaven GM, Ida Chen Y-D, Jeppesen J, Maheux P, Krauss RM: Insulin resistance and hyperinsulinemia in individuals with small, dense, low density lipoprotein particles. J Clin Invest 1993; 92: 141-146.

12 Austin MA, Brunzell JD, Fitch WL, Krauss RM: Inheritance of low density lipoprotein subclass patterns in familial combined hyperlipidemia. Arteriosclerosis 1990; 10: 520-530.

13 Bredie SJH, Kiemeney LA, de Haan AFJ, Demacker PNM, Stalenhoef AFH: Inherited suspectibility determines the distribution of dense low-density lipoprotein subfraction profiles in familial combined hyperlipidemia. Am J Hum Genet 1996; 58: 812-822.

14 Edwards KL, Mahaney MC, Motulsky AG, Austin MA: Pleiotropic genetic effects on LDL size, plasma triglyceride, and HDL cholesterol in families. Arterioscler Thromb Vasc Biol 1999; 19: 24562464.

15 Austin MA, King MC, Vranizan KM, Krauss RM: Atherogenic lipoprotein phenotype. A proposed genetic marker for coronary heart disease risk. Circulation 1990; 82: 495-506.

16 Porkka KV, Viikari JS, Rönnemaa T, Marniemi J, Åkerblom HK: Age and gender specific serum lipid and apolipoprotein fractiles of Finnish children and young adults. The Cardiovascular Risk in Young Finns Study. Acta Paediatr 1994; 83: 838-848.

17 Vartiainen P, Puska P, Jousilahti P, Korhonen HJ, Tuomilehto J, Nissinen A: Twenty-year trends in coronary risk factors in north Karelia and in other areas of Finland. Int J Epidemiol 1994; 23: 495-504.

18 Vakkilainen J, Jauhiainen M, Ylitalo K et al: LDL particle size in familial combined hyperlipidemia. Effects of serum lipids, lipoprotein-modifying enzymes, and lipid transfer proteins. J Lipid Res 2002; 43: 598-603.

19 Vakkilainen J, Porkka KVK, Nuotio I et al: Glucose intolerance in familial combined hyperlipidaemia. Eur J Clin Invest 1998; 28: $24-32$.

20 Ylitalo K, Pajukanta P, Meri S et al: Serum C3 but not plasma acylation-stimulating protein is elevated in Finnish patients with familial combined hyperlipidemia. Arterioscler Thromb Vasc Biol 2001; $21: 838-843$.
21 S.A.G.E.: Statistical analysis for genetic epidemiology, Version 4.0 Beta 6, 2000. Case Western Reserve University, Cleveland, 2000.

22 S.A.G.E.: Statistical analysis for genetic epidemiology, Version 3.1 Beta 6, 1997. Case Western Reserve University, Cleveland, 1997.

23 Bonney GE: On the statistical determination of major gene mechanisms in continuous human traits: regressive models. Am J Hum Genet 1984; 18: 731-749.

24 Akaike J: A new look at the statistical model identification. IEEE Transautomat Control 1974, AC-19: 716-729.

25 de la Chapelle A: Disease gene mapping in isolated human populations: the example of Finland. J Med Genet 1993; 30: 857-865.

26 Packard CJ, Shepherd J: Lipoprotein heterogeneity and apolipoprotein B metabolism. Arterioscler Thromb Vasc Biol 1997; 17: $3542-3556$

27 Jarvik GP: Complex segregation analyses: uses and limitations. Am J Hum Genet 1998; 63: 942-946.

28 Veerkamp MJ, de Graaf J, Bredie SJ, Hendriks JC, Demacker PN, Stalenhoef AF: Diagnosis of familial combined hyperlipidemia based on lipid phenotype expression in 32 families: results of a 5-year follow-up study. Arterioscler Thromb Vasc Biol 2002; 22 $274-282$.

29 Cullen P, Farren B, Scott J, Farrall M: Complex segregation analysis provides evidence for a major gene acting on serum triglyceride levels in 55 British families with familial combined hyperlipidemia. Arterioscler Thromb 1994; 14: 1233-1249.

30 Jarvik GP, Brunzell JD, Austin MA, Krauss RM, Motulsky A, Wijsman E: Genetic predictors of FCHL in four large pedigrees. Influence of apoB level major locus predicted genotype and LDL subclass phenotype. Arterioscler Thromb 1994; 14: 16871694.

31 Allayee H, Aouizerat BE, Cantor RM et al: Families with familial combined hyperlipidemia and families enriched for coronary artery disease share genetic determinants for the atherogenic phenotype. Am J Hum Genet 1998; 63: 577-585.

32 Austin MA, King MC, Vranizan KM, Newman B, Krauss RM: Inheritance of low-density lipoprotein subclass patterns: results of complex segregation analysis. Am J Hum Genet 1988; 43 $838-846$.

33 Austin MA, Jarvik GP, Hokanson JE, Edwards K: Complex segregation analysis of LDL peak particle diameter. Genet Epidemiol 1993; 10: $599-604$.

34 Juo SHH, Bredie SJH, Kiemeney LA, Demacker PNM, Stalenhoef AFH: A common genetic mechanism determines plasma apolipoprotein $\mathrm{B}$ levels and dense LDL subfraction distribution in familial combined hyperlipidemia. Am J Hum Genet 1998; 63: $586-594$

35 Hokanson JE, Austin MA, Zambon A, Brunzell JD: Plasma triglyceride and LDL heterogeneity in familial combined hyperlipidemia. Arterioscler Thromb 1993; 13: 427-434. 